\title{
Impact of $\mathbf{T}_{\mathrm{e}}$ and $\mathbf{n}_{\mathrm{e}}$ on edge current density profiles in ELM mitigated regimes on ASDEX Upgrade
}

\author{
M.G. Dunne ${ }^{1}$, S. Rathgeber ${ }^{1}$, A. Burckhart ${ }^{1}$, R. Fischer ${ }^{1}$, L. \\ Giannone $^{1}$, P.J. McCarthy ${ }^{2}$, P.A. Schneider ${ }^{1}$, E. Wolfrum ${ }^{1}$, the \\ ASDEX Upgrade Team ${ }^{1}$ \\ ${ }^{1}$ Max-Planck-Institut für Plasmaphysik, Boltzmannstraße 2, D-85748 \\ Garching,Germany \\ ${ }^{2}$ Department of Physics, University College Cork, Association Euratom-DCU, Cork, \\ Ireland \\ E-mail: michael.dunne@ipp.mpg.de
}

\begin{abstract}
ELM resolved edge current density profiles are reconstructed using the CLISTE equilibrium code. As input, highly spatially and temporally resolved edge electron temperature and density profiles are used in addition to data from the extensive set of external poloidal field measurements available at ASDEX Upgrade, flux loop difference measurements, and current measurements in the scrape-off layer. Both the local and flux surface averaged current density profiles are analysed for several ELM mitigation regimes. The focus throughout is on the impact of altered temperature and density profiles on the current density. In particular, many ELM mitigation regimes rely on operation at high density. Two reference plasmas with type-I ELMs are analysed, one with a deuterium gas puff and one without, in order to provide a reference for the behaviour in Type-II ELMy regimes and high density ELM mitigation with external magnetic perturbations at ASDEX Upgrade. For Type-II ELMs it is found that while a similar pedestal top pressure is sustained at the higher density, the temperature gradient decreases in the pedestal. This results in lower local and flux surface averaged current densities in these phases, which reduces the drive for the peeling mode. No significant differences between the current density measured in the Type-I phase and ELM mitigated phase is seen when external perturbations are applied, though the pedestal top density was increased. Finally, ELMs during the nitrogen seeded phase of a high performance discharge are analysed and compared to ELMs in the reference phase. An increased pedestal pressure gradient, which is the source of confinement improvement in impurity seeded discharges, causes a local current density increase. However, the increased $\mathrm{Z}_{\text {eff }}$ in the pedestal acts to reduce the flux surface averaged current density. This dichotomy, which is not observed in other mitigation regimes, could act to stabilise both the ballooning mode and the peeling mode at the same time.
\end{abstract}




\section{Introduction}

The foreseen operational scenario for the ITER tokamak, which is currently under construction in Cadarache, France, is the H-mode. This scenario offers operation at a high level of confinement which is essential to the success of fusion as an energy source. The H-mode derives its high confinement properties from a transport barrier which forms at the edge (outer $\sim 2 \mathrm{~cm}$ ) of the plasma which is characterised by steep gradients in both temperature and density profiles. These steep edge gradients are the source of the improved confinement in H-mode plasmas. However, this confinement does not come without a price; the steep gradients provide a source of free energy for ballooning modes which are linked to the occurence of edge localised modes (ELMs). These MHD modes release $\sim 10 \%$ of the plasma stored energy in a short timescale (of the order of one millisecond) as an abrupt crash, lowering the gradients in the edge transport barrier, known as the pedestal, and expelling a fraction of the confined plasma. The expelled particles and heat then travel along the open field lines in the unconfined plasma and impact the first wall materials, depositing a large amount of heat on the divertor tiles. While this sudden heat deposition does not pose issues in present-day tokamak devices, ELM induced heat loads are likely to be intolerable in larger future devices, such as ITER[1].

Due to the necessity to operate at high confinement, yet without ELMs, several methods of ELM mitigation are being intensively researched. There are two principle lines of investigation: plasma scenario based and external perturbations of the plasma. A prominent example of the former category are Type-II ELMs[2, 3, 4], where the plasma shape is changed from single null to an almost double null configuration and a high external gas puff is applied to increase the density. Several options for external ELM mitigation are being investigated, such as using frozen deuterium pellets to trigger an $\operatorname{ELM}[5,6]$, vertical "kicks" of the plasma[7, 8, 9, 5], supersonic molecular beam injection[10, 11], and external magnetic perturbations[12, 13, 14, 4]. The eventual goal of this line of research is a set of operational scenarios that retain the high confinement of the H-mode while mitigating ELM losses. There are both experimental and theory based approaches to this. Scenario development at several tokamaks has uncovered many operational regimes with reduced ELM losses, such as type-II ELMs[2]. Advances in MHD theory help to shed light on the ELM trigger mechanism.

The currently favoured theory to explain ELMs is peeling-ballooning theory[15], which posits a critical edge pressure gradient and current density as the ELM trigger. More specifically, the critical edge pressure gradient is also dependent on the pedestal width; at a constant pressure gradient, the pedestal becomes more unstable at higher pedestal widths. This is the basis behind the EPED model of the ELM cycle[16] and explains the observed pressure gradient saturation before the ELM crash[17], assuming that the pedestal width does grow towards the ELM cycle. This has recently been reported to be the case at MAST[18]. In addition to the pressure gradient, two aspects of the current density are thought to characterise the drive it provides to the peeling mode; 
its peak height and its value at the separatrix. In a systematic study of the effect various changes to the pressure and current density profiles have on a reference equilibrium for ITER[19] it was shown that an increase of the current density at the separatrix can lower the stability of the pedestal to external kink modes. However, stability analysis using the JOREK code[20] has shown that true x-point geometry, which is not typically included in linear ideal MHD analysis, stabilises traditional peeling modes, but destabilises a peeling-tearing mode which can be approximated at low resistivity as a peeling mode by ideal MHD codes. As such, it is unclear what impact a finite current density at the separatrix has on pedestal stability, assuming all other factors are kept constant. In general, however, an increased current density peak in the pedestal region does lead to more unstable peeling modes.

The edge pressure gradient has been well diagnosed in many ELM mitigation scenarios, but measurements of the edge current density have only recently become available. The measurements from DIII-D[21], MAST[22], and ASDEX-Upgrade[23] have all shown that the pre-ELM current density in the pedestal is consistent with a neoclassical nature, i.e. it is driven by gradients in the temperature and density profiles. The form of the edge current density profile also depends on the collisionality in the pedestal. Sauter et al.[24, 25] have derived an analytical fit to determine the neoclassical current density for arbitrary collisionality and plasma shape. Since many ELM mitigation scenarios rely on changing the collisionality in the pedestal, it is possible that the current density could be a major factor which determines access to this regimes.

This paper will analyse the edge current density for a number of ELM mitigation scenarios on the ASDEX Upgrade (AUG) tokamak with a focus on the effect of altered temperature and density profiles on the current density profile as the plasma moves from a type-I ELMy scenario to an ELM mitigated scenario. The edge current density will be analysed using both the CLISTE equilibrium code[26, 27], taking into account edge pressure profiles, external magnetic measurements, and scrape-off layer current measurements and compared with the predictions from neoclassical theory. Since high confinement is desired for these scenarios, there is a requirement that the pressure gradients remain high; altering the edge current density profile such that it either stabilises the ballooning modes, or such that the peeling component of ELMs is reduced or eliminated are possible mechanisms for ELM mitigation. The rest of the paper falls into four sections. Section 2 compares two improved H-mode scenario discharges in AUG, one with and one without a deuterium gas puff in the main part of the discharge. These two discharges have the same pedestal top pressure, but different temperature and density profiles, serving as a basis for comparison for the later sections. Section 3 analyses the current density in a type-II ELMy scenario. Section 4 details the changes the edge current density profile undergoes as the plasma transitions from a type-I ELMy to a mitigated ELM regime in the presence of external magnetic perturbations. The current density profiles in discharges where nitrogen gas is puffed such that nitrogen appears as a trace impurity $\left(\mathrm{C}_{\mathrm{N}} \sim 1-2 \%\right)$ are analysed. A distinction between gas fuelling and impurity seeding is assumed for the content of this paper, where fuelling 
refers to the main ion gas (deuterium in all cases here) and seeding refers to the puffing of, in this case, nitrogen, in section 5. A summary and comparison of the results from each of these sections along with a conclusion is given in section 6 .

\section{Type-I ELMs}

Before investigating the impact of temperature and density profile modifications on the current density during ELM mitigated regimes, it is useful to analyse what happens to the current density when these profiles change during normal type-I regimes. A well diagnosed discharge pair, AUG \#23221 and AUG \#23222 have been used for this purpose. Both discharges featured 7.5 MW of NBI heating power, 1.3 MW of ECRH, a 1 MA plasma current, -2.5 T magnetic field, and an average triangularity of 0.25. Both discharges differed in the amount of gas fuelling applied; discharge \#23221 had a gas puff rate of $6 \times 10^{21}$ electrons s ${ }^{-1}$, while $\# 23222$ had no gas puff during the main part of the discharge. Time traces of the key plasma parameters are shown in figure 1 with (a) applied heating power and the radiated power in both discharges, (b) the plasma stored energies, (c) gas puff rates, (d) line integrated densities, (e) pedestal top temperature, and (f) Type-I ELM frequency. The pedestal top temperature in this figure (and the timetraces in the following sections, unless otherwise stated) has been determined by using a two line fitting method[28]. While the temperature and density signals are significantly different in the two discharges, the pedestal top pressure is similar. The higher ELM frequency in the higher heating power phase of the fuelled discharge is clearly seen in this timetrace, indicating smaller ELMs.

The pre- and post-ELM edge electron temperature and density profiles for these discharges are shown in figure 2. The solid lines indicate pre-ELM timepoints, while the dashed lines are the post-ELM profiles. The red profiles are from discharge \#23221 and the blue ones are from discharge \#23222. The profile fits are made using a modified tanh function, which describes the profiles as a combination of a simple tanh with additional polynomials for the core and scrape-off layer (SOL) plasmas. The tanh function is also used to define the critical pedestal parameters, notably the pedestal top, pedestal bottom, the half width, and the mid-point of the pedestal. These profiles were made by mapping the raw data to a normalised flux radius and applying a least squares fit using the modified tanh as the fit function. In this case, $\rho_{\text {poloidal }}$, defined as

$$
\rho_{\text {poloidal }}=\sqrt{\frac{\psi_{\text {axis }}-\psi}{\psi_{\text {axis }}-\psi_{\text {sep }}}}
$$

The error bars shown in this figure correspond to a $1 \sigma$ scatter in the raw data; the electron density uncertainties stop near the pedestal top as no core TS data were available for these discharges. However, the core profiles are additionally constrained by line integrated density measurements.

The raw data for temperature were taken from an electron cyclotron emission (ECE) diagnostic[29] and a Thomson Scattering (TS) diagnostic[30, 31]. The density data were 


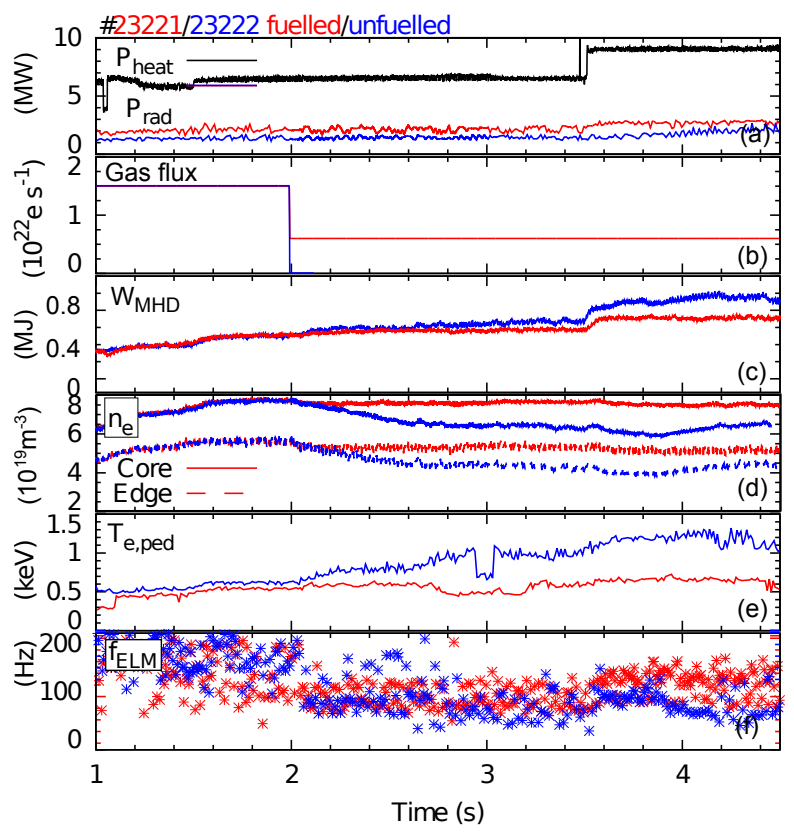

Figure 1. Time traces for discharges \#23221 (red) and \#23222 (blue) of (a) heating power (black) and radiated power, (b) plasma stored energy, (c) fuelling rate, (d) core (solid) and edge (dashed) line integrated density, (e) pedestal top temperature, (f) ELM frequency. The higher fuelling rate in discharge \#23221 leads to lower confinement, indicated by the lower pedestal top temperature and plasma stored energy.

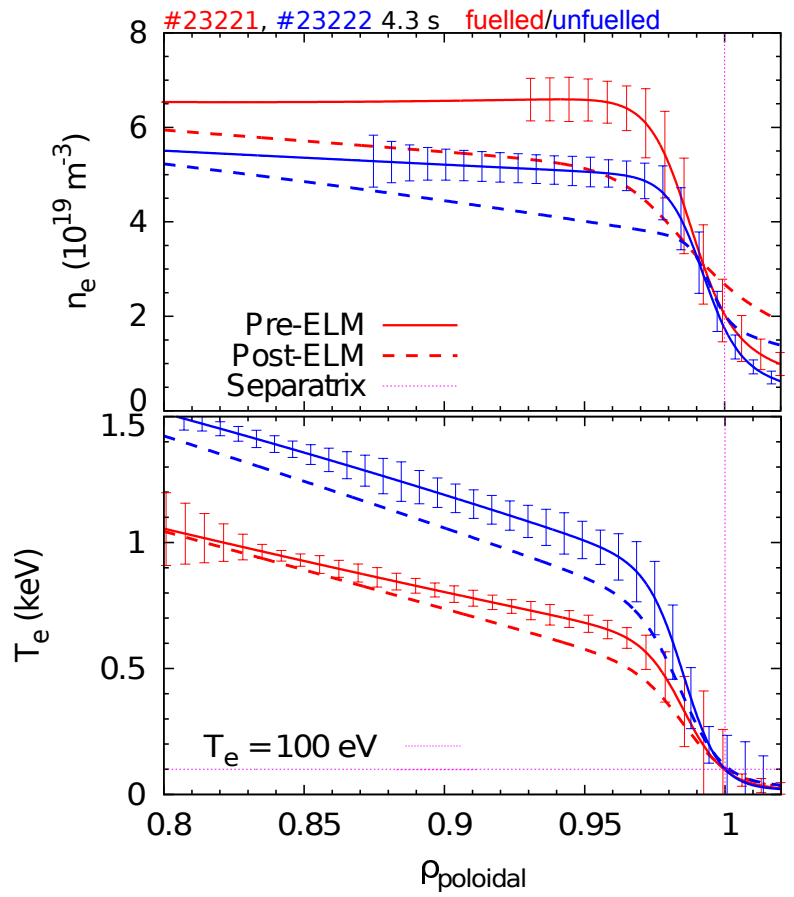

Figure 2. (a) electron density profiles for discharges \#23221 and \#23222 showing pre-ELM (solid) and post-ELM (dashed) profiles. (b) electron temperature profiles with the same colour and line styles. The conservation of the temperature gradient after the ELM crash is evident in discharge \#23222, despite the larger ELM crash. 
taken from a lithium beam emission spectroscopy (LiBES) diagnostic [32, 33], TS, and 5 lines of a deuterium-cyanide-nitrogen (DCN) inteferometry diagnostic[34] to provide line integrated density measurements. The inclusion of TS data in both cases allows an intrinsic alignment between the temperature and density profiles. For inclusion into CLISTE, the fitted profiles were interpolated onto a 30 -point grid in $\mathrm{R}_{\text {major }}$, a real space coordinate along a horizontal radial line passing through the magnetic axis. Since CLISTE requires the total pressure profile, the pedestal ion temperatures were assumed to be the same as the electron temperatures, which is a reasonable assumption given the collisionality range in which AUG typically operates[35]. The ion density was calculated in this case as a function of the electron density, assuming quasi-neutrality, an effective charge of 1.9, and carbon as a dominant impurity. Fast ions were ignored as they typically play only a small role at the pedestal top.

The pre-ELM profiles shown in figure 2 are fitted to data taken between $3-0.5 \mathrm{~ms}$ before the ELM crash, while the post-ELM profiles were taken between $1-2.5 \mathrm{~ms}$ after the crash. The ELM losses for discharge \#23221 amount to almost $40 \mathrm{kPa}\left(5.5 \% \mathrm{~W}_{\mathrm{MHD}}\right)$ while the losses in discharge \#23222 are larger with an absolute magnitude of $\sim 50 \mathrm{kPa}$ $\left(6.5 \% \mathrm{~W}_{\mathrm{MHD}}\right)$. This can also be seen in the divertor current measurements in figure 3, which shows the evolution of the current measured via a shunt at the inner divertor. Again, the red corresponds to discharge 23221, the blue to 23222. The error bars in this figure indicate one standard deviation of the data. The divertor currents have been shown to be driven by a thermocurrent in the inter-ELM period on AUG[36], while a comparison of modelling with experiment during ELMs on the TCV tokamak[37] showed that the SOL currents during the ELM crash in this case were also of a predominantly thermocurrent nature. The magnitude of the divertor currents is then proportional to the difference between the temperatures at the inner and outer divertors and the SOL temperature. The temperature and density profiles from figure 2 were used as input for

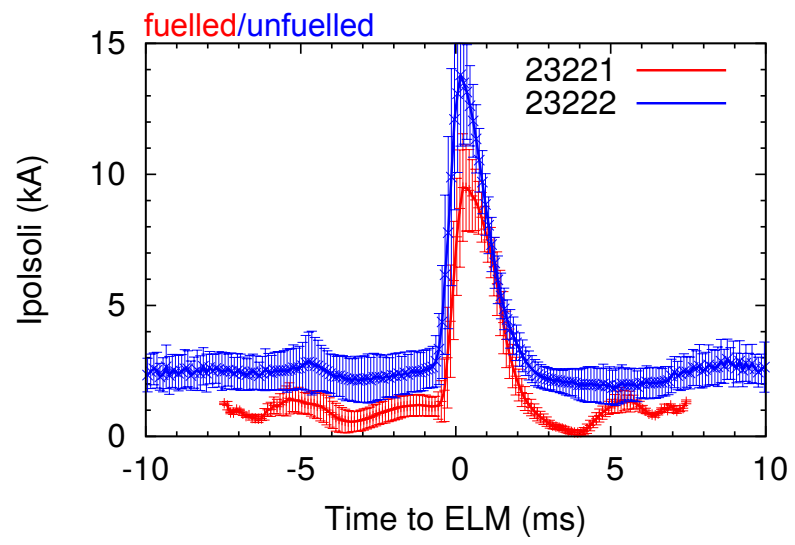

Figure 3. Divertor currents for discharges \#23221 (red) and \#23222 (blue) relative to the ELM crash. The error bars indicate the one sigma spread of the data. This signal can be used as an indicator for the size of the ELM crash, showing the larger magnitude of the crash in the unfuelled discharge \#23222.

the CLISTE code. The external magnetic signals were averaged during the same time 


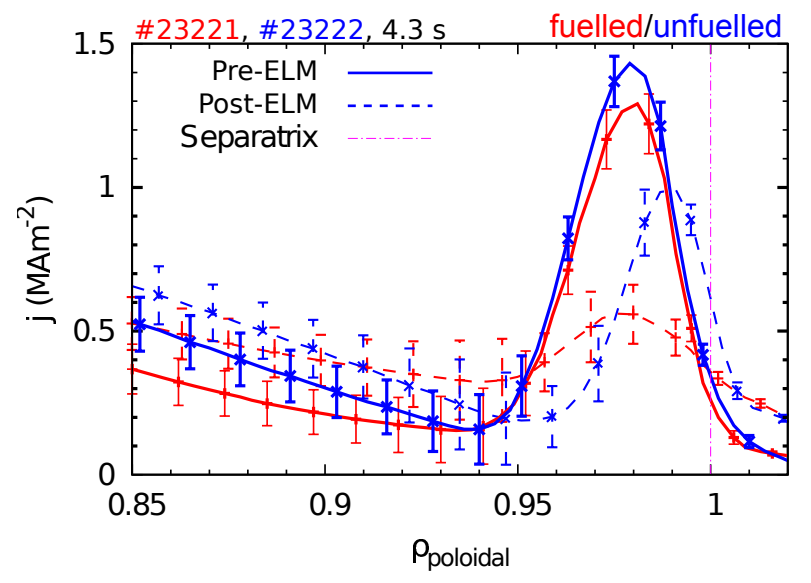

Figure 4. Pre-ELM (solid) and post-ELM (dashed) profiles of the local current density at the LFS midplane determined using the CLISTE code. The error bars indicate one sigma confidence bands, as determined by CLISTE. Notable is the presence of a strong current density peak after the ELM crash in discharge \#23222, as well as the higher pre-ELM peak magnitude.

windows as the kinetic data.

The local edge current density for both discharges is shown in figure 4, with the same colour and line key as in figure 2. The error bars shown indicate $1 \sigma$ confidence bands, as calculated by CLISTE. The impact of including the divertor current measurements on the accuracy of the profile reconstruction can be seen in the SOL portion of the fit. Here, the uncertainties calculated by CLISTE are much smaller than inside the confined plasma, indicating that the current density profile in this region is well determined by the equilibrium solver. The pre-ELM profiles are similar, with the peak edge current density occuring at the same location in both. In discharge \#23222 the peak is higher, though this is at the limit of what can be determined with the given confidence intervals. The post-ELM profiles differ significantly, with the peak in the case of \#23221 almost completely removed by the ELM. There is still a significant peak in discharge \#23222 after the ELM crash; this is due to the edge density gradient remaining relatively unaffected by the ELM in this discharge, as well as a smaller decrease in edge temperature gradient, which means significant Pfirsch-Schlüter and bootstrap currents are still present after the ELM.

The flux-surface averaged parallel current density profiles show similar characteristics to the local current density. Before the ELM crash, shown in figure 5(a), there are again differences in the magnitudes of the current density peaks. The solid lines show the CLISTE fitted current densities, with associated $1 \sigma$ confidence intervals, while the dashed lines with boxes show the current density as calculated using the formulae from Sauter et al.[24, 25]. This neoclassical current density contains contributions from both the bootstrap current as well as the ohmic current. Both CLISTE and the neoclassical current density show that discharge \#23222 should have a slightly higher edge current density. The difference in this case, however, is approximately the same magnitude 
as the uncertainties calculated by CLISTE. The post-ELM profiles also demonstrate a similar phenomenology as the local current density profiles. The current density peak in discharge \#23221 almost completely disappears, while there is still a significant bootstrap current driven in discharge \#23222, due to the conserved electron temperature gradient in the outer region of the pedestal. The neoclassically driven current in the pedestal is supplemented by a large parallel current density driven in the SOL as a result of thermocurrents from the asymmetric ELM induced heat loads.

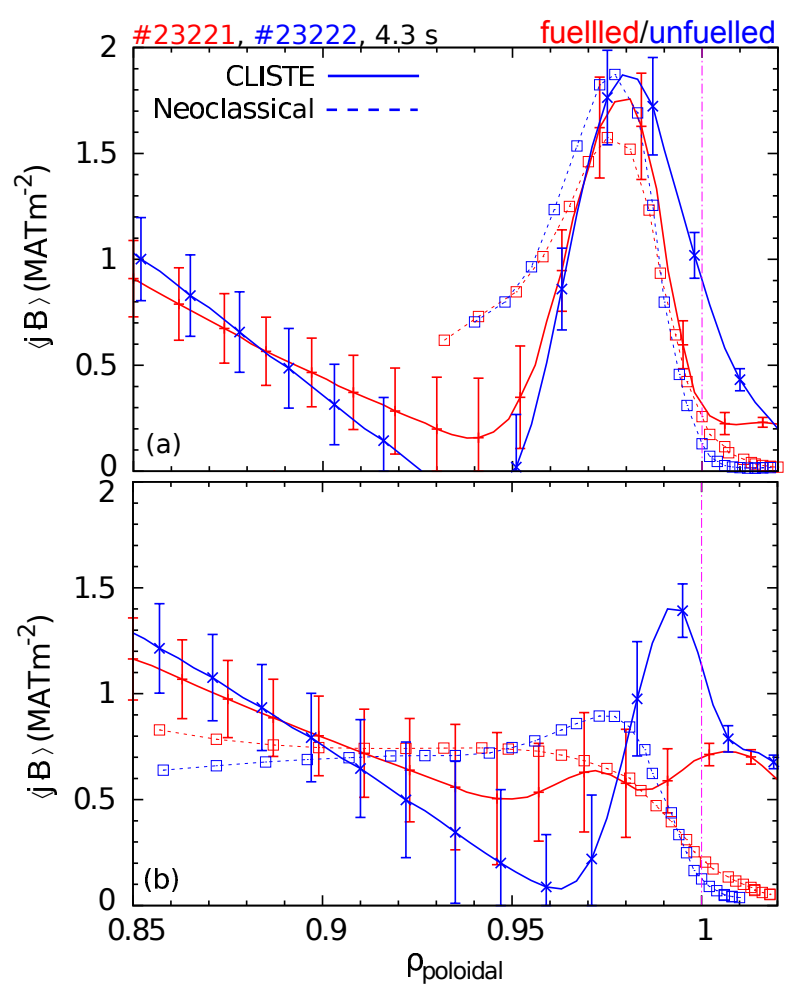

Figure 5. (a) pre-ELM profiles of $\langle\boldsymbol{j} \cdot \boldsymbol{B}\rangle$ from CLISTE (solid) and neoclassical calculations (dashed with boxes) for discharges \#23221 (red) and \#23222 (blue). (b) post-ELM profiles corresponding to the profiles shown in (a). Again, a slight increase in the magnitude of the edge current density is visible, as is a significant edge peak after the ELM crash in the unfuelled case (\#23222)

Despite the significantly different pedestal top collisionality in both discharges, the dominant factor which determines the shape and magnitude of both the local and flux surface averaged edge current density profiles appears to be the pressure profile. It seems unlikely that the small increase in the local peak edge current density in the unfuelled case could be responsible for the ELM crash. The larger current density at the separatrix does appear to be linked with an increased current in the SOL plasma; while this may be related to the change in temperature and density profiles in the pedestal, it is a higher order effect and thus requires detailed modelling to determine its impact. The higher flux surface averaged current density at the separatrix in the unfuelled case could indicate an increased drive for a peeling mode. There are, however, no studies showing any conclusive link between the type of unstable mode, or the width of its 
poloidal mode number spectrum on the size of the ELM crash. The phenomenology of increasing density (or collisionality) leading to smaller ELMs[38] which is observed in this section is emphasised even more in the following section on type-II ELMs, where it is also accompanied by a change in the plasma shape. However, based on the results in this section, it is unlikely that the finding by Loarte et al.[38] is due to a direct change in the current density profile from the temperature and density profiles.

\section{Type-II ELMs}

Type-II ELMs are quite different in structure to type-I ELMs. They occur with a high frequency (several hundred $\mathrm{Hz}$ ) and exhibit a "grassy" nature. A broadband electron temperature fluctuation is also associated with this regime, occuring in a frequency range between $20-65 \mathrm{kHz}$ in an off-midplane location radially inside the pedestal top[39]. The entry conditions to type-II ELMy H-modes have been well documented[2, 3, 4, 40]. The requirements are a high plasma density and an almost connected double null shaping. These are high performance plasmas; the discharge studied here, AUG\#25740, has a $\mathrm{H}$-factor of 1 and a plasma stored energy equal to that during the type-I ELMy phase, while keeping the same normalised beta of 1.7 at a plasma current of $800 \mathrm{kA}$ and central toroidal magnetic field of $-2.5 \mathrm{~T}$. The average triangularity is changed from $\sim 0.36$ to $\sim 0.38$ as the plasma configuration is varied from lower single null to an almost double null shape. Timetraces of (a) the heating power, radiated power, (b) gas fuelling rate, (c) plasma stored energy, (d) core and edge plasma densities, (e) pedestal top temperature, and (f) Type-I ELM frequency are shown in figure 6. Type-II ELMs are present in the discharge between 2.5 and $3.7 \mathrm{~s}$, as indicated by lack of large fluctuations in the plasma stored energy and edge density time traces and the frequency of the Type-I ELMs going to zero. In order to increase the density to the level required for type-II ELMs a large gas ramp is applied and held constant. A back transition to a type-I ELMing regime is effected by ramping down the gas fuelling at $3 \mathrm{~s}$. During the type-II phase, both the core and edge densities are increased, while the pedestal top temperature is decreased, reaching a minimum as the gas fuelling begins to ramp down. The temperature slowly recovers as the density decreases and clear type-I ELMs are again observed after $3.7 \mathrm{~s}$.

Profiles of temperature and density for this discharge are shown in figure 7 . The type-I profiles are taken from the timeslice between 1.7 and $2.2 \mathrm{~s}$ and averaged over many ELMs between 3 and $0.5 \mathrm{~ms}$ prior to an ELM crash, while the type-II profiles are averaged over a $200 \mathrm{~ms}$ timeslice between 2.75 and $2.95 \mathrm{~s}$. These timepoints have also been analysed by Wolfrum et al.[40], and so shall be discussed only briefly here. Moving from the type-I to the type-II ELMs, the density pedestal top increases, while the gradient remains almost constant, implying that the same gradient clamping mechanisms for the density profile are present in both ELMy phases. The temperature pedestal decreases in height and is also wider, leading to a significantly reduced gradient. However, the combined pressure profile has a similar pedestal top value, albeit with a larger pedestal width and, hence, a lower gradient. Due to the higher collisionality 


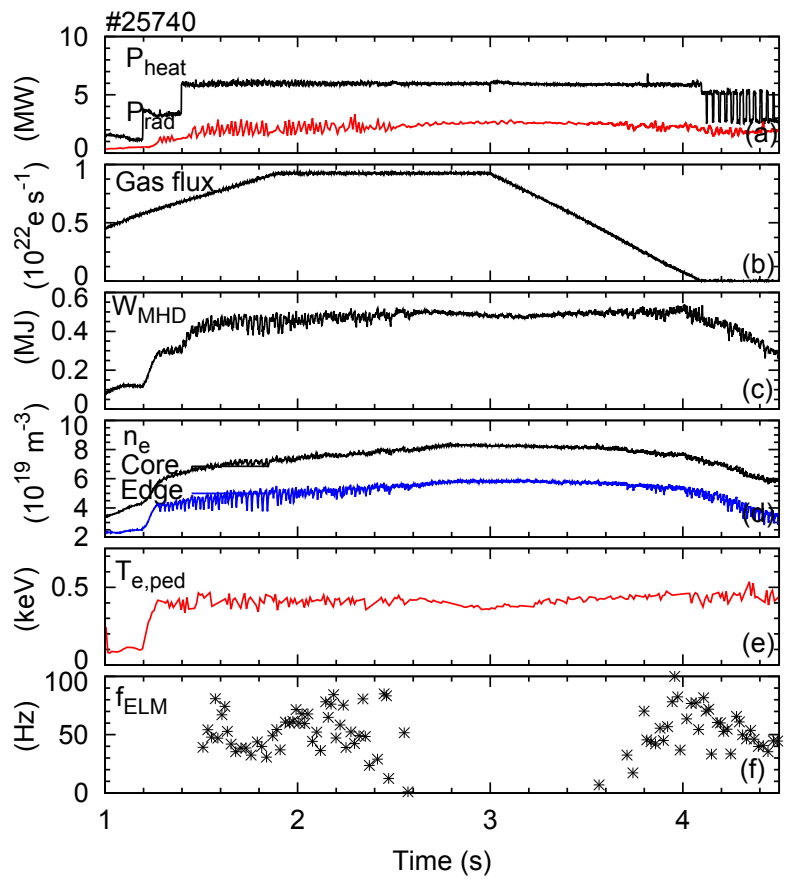

Figure 6. Time traces of (a) heating power (black), radiated power (red), (b) gas fuelling rate, (c) plasma stored energy, (d) core (black) and edge (blue) line integrated densities, (e) pedestal top temperature, and (f) Type-I ELM frequency for discharge \#25740. The plasma enters a type-II ELMing regime at $2.5 \mathrm{~s}$ and re-enters a type-I regime after the gas fuelling is ramped down at $3.7 \mathrm{~s}$.

and lower temperature gradient, it can be expected that moving into the type-II ELMy regime would be accompanied by a lower edge current density.

The profiles shown in figure 7 were used as input for CLISTE, with the magnetic data also averaged in the same time windows. The post-ELM profiles from the type-I case were not reconstructed in this case. The local LFS profiles are shown in figure 8(a) for these two timepoints with corresponding $1 \sigma$ confidence bands. As expected, the local current density is indeed lower in the type-II ELMy case than in the type-I case, while the current density peak is approximately $30 \%$ broader, as determined from a Gaussian fit to the respective peaks. The separatrix and SOL values remain the same, despite an increase in the measured SOL currents. Figure 8(b) shows the measured (solid lines) and neoclassical (dashed lines with boxes) flux surface averaged parallel current densities. This measure of the current density also decreases when moving into the type-II regime, though the width of the peak decreases by $20 \%$. In the pre-ELM case, there is excellent agreement between CLISTE and the neoclassical calculation, with the neoclassical calculation showing a slightly more peaked current density profile. For the type-II case, the agreement is not quite as good, though still within the uncertainties of the CLISTE fit. While the neoclassically calculated shape and peak location agree with CLISTE in the type-I case, the calculated type-II peak is further inside the plasma and also broader.

The reduction in the local current density is due to the lower pressure gradient in 


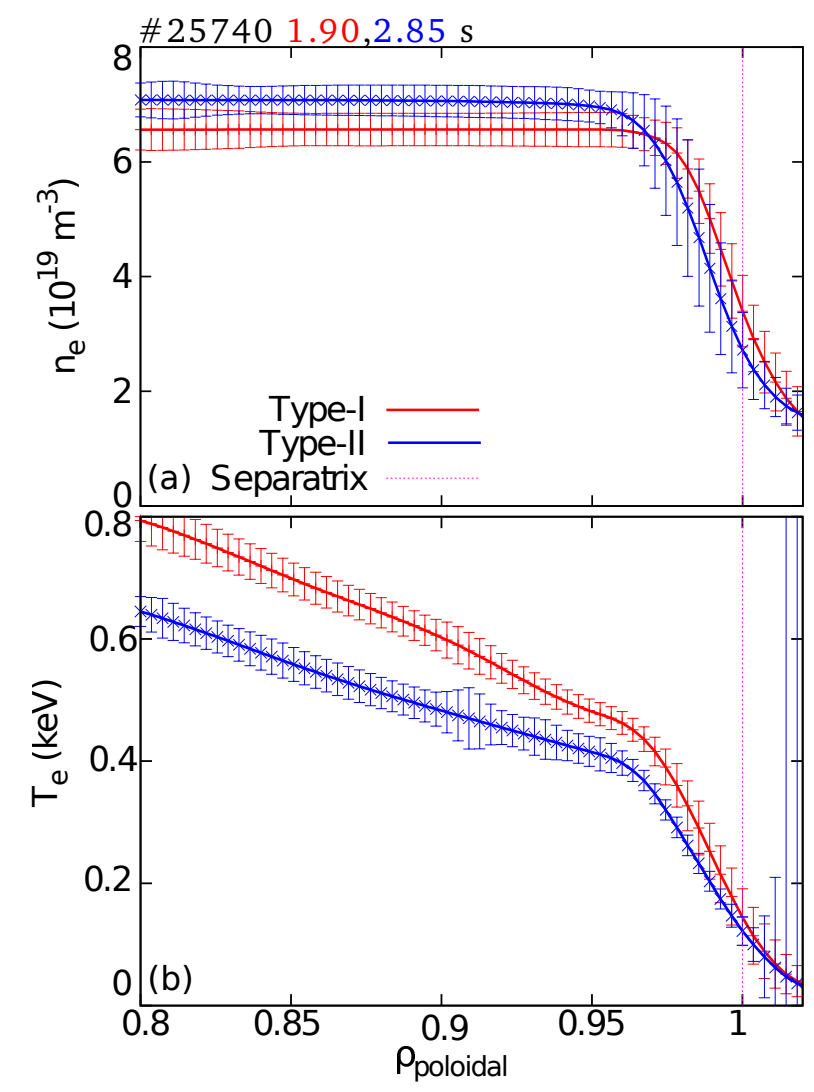

Figure 7. (a) electron density profiles for pre type-I ELM (red) and type-II ELMy (blue) phases in discharge \#25740. (b) electron temperature profiles corresponding to the density profiles in (a). While the density gradient remains the same in the pedestal during both phases, the temperature gradient drops.

the type-II phase, while the reduced parallel current density is due to a combination of the higher collisionality and lower electron temperature gradient in the pedestal. However, the increased density and fuelling rates are not the only requirements for entering the type-II phase; the plasma shape must also approach double null which also acts to increase the magnetic shear. Saarelma et al.[41] have hypothesised that it is only with the combination of these two parameters that the shear at the plasma edge reaches a sufficient value to block access to the "second stability" region. This then means that the pedestal is dominated by $n=\infty$ ballooning modes, which could act as a soft boundary, explaining the small type-II ELMs. However, there is another scenario at AUG which also features small ELMs which are similar in magnitude to type-II ELMs but are observed in lower triangularity discharges; the ELMs seen during high density discharges when external magnetic perturbations are applied. These will be analysed in the following section. 


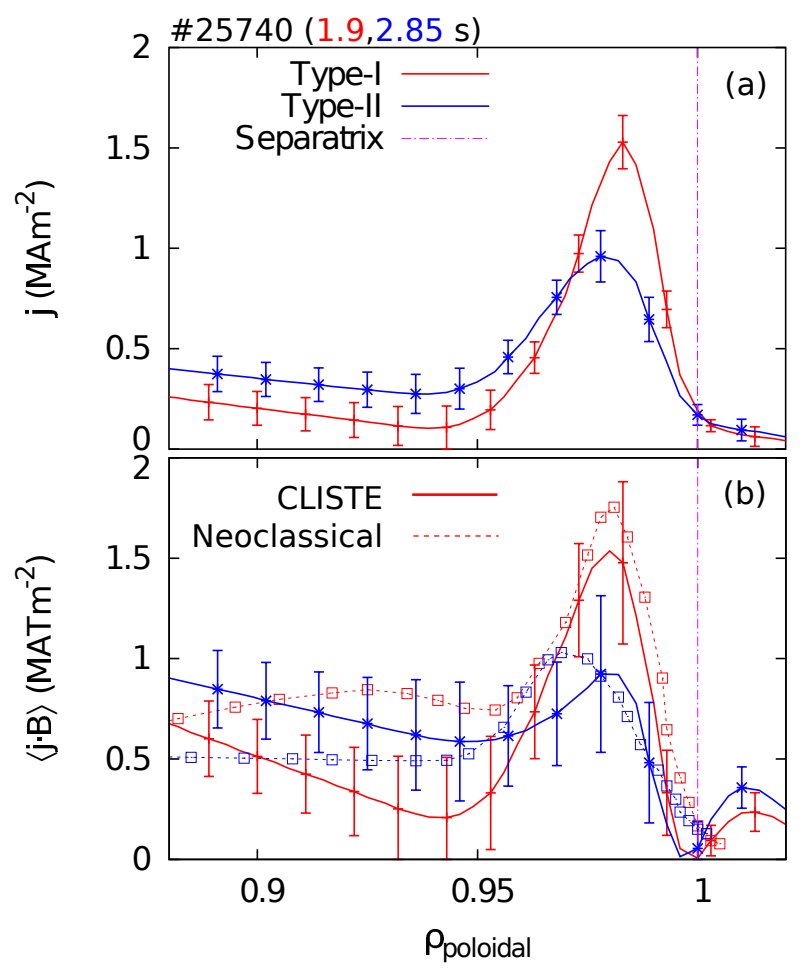

Figure 8. (a): local current density profiles for the type-I (red) and type-II (blue) ELMy phases of discharge \#25740, showing a signifcantly reduced current density profile in the type-II phase. (b) $\langle\boldsymbol{j} \cdot \boldsymbol{B}\rangle$ profiles from CLISTE (solid) and neoclassical predictions (dashed with boxes). This flux surface averaged current density also shows a significant decrease in the type-II ELMy phase compared to the pre-type-I ELM profile.

\section{ELM mitigation via external magnetic perturbations at high density}

External magnetic perturbations have been applied on several tokamaks in order to achieve mitigated ELM types[13, 12, 42, 14]. On the DIII-D tokamak, full ELM suppression is obtained at low densities[43] while high collisionality ELM mitigation is also achieved[12]. On AUG, magnetic perturbation coils are arranged in two rows of 8 coils each. These coils are mounted inside the vacuum vessel, with one row above and one row below the magnetic midplane. This arrangement allows perturbations with mode numbers of 1,2 , and 4 , in even or odd parity to be used. All of these combinations have been used to mitigate ELMs[44]. However, simply applying the perturbation coils is not enough at AUG; a density threshold has also been observed $[45,46]$.

A discharge featuring odd parity magnetic perturbations (\#28847) was analysed to determine any changes in the edge current density as the plasma transitions from a type-I ELMy phase to a mitigated ELM regime. This discharge had an $800 \mathrm{kA}$ plasma current, $-2.5 \mathrm{~T}$ magnetic field, and average triangularity of 0.28 . Figure 9 shows (a) the heating (black) and radiated (red) power, which are constant throughout the discharge, (b) the deuterium fuelling rate, which is ramped slowly until $5 \mathrm{~s}$ when the ramp rate is further increased, (c) the plasma stored energy, (d) the core (black) and 


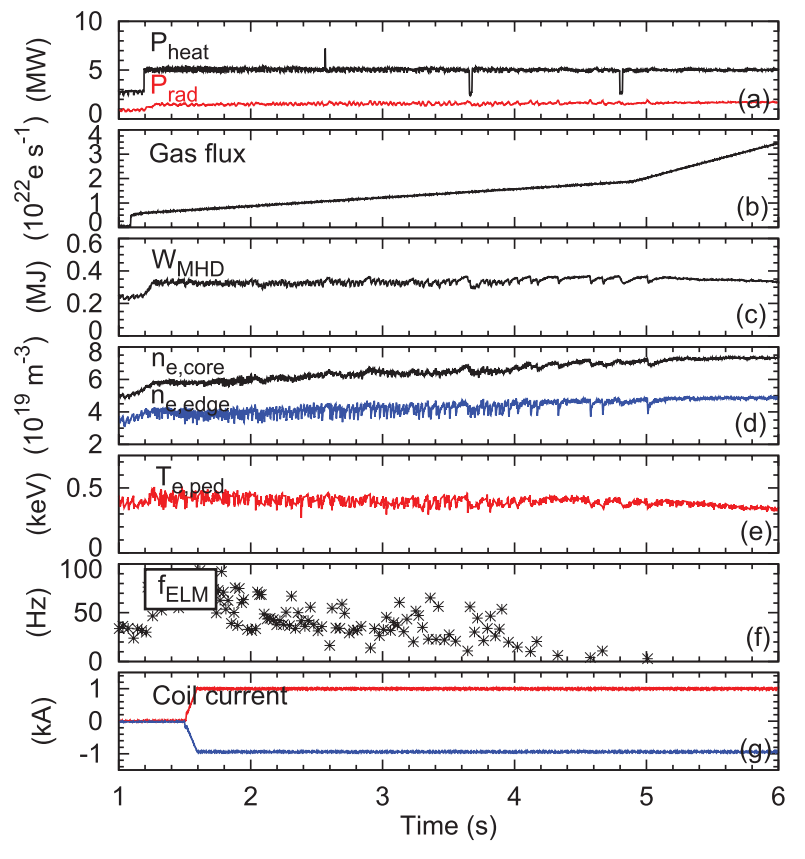

Figure 9. Timetraces of (a) heating power (black), radiated power (red), (b) gas fuelling rate, (c) plasma stored energy, (d) core (black) and edge (blue) line integrated densities, (e) temperature near the pedestal top, (f) Type-I ELM frequency, and (g) time trace of magnetic perturbation coil currents for discharge \#28847. The density increases slowly throughout the discharge, with the temperature decreasing only slightly until the large gas fuelling ramp at $5 \mathrm{~s}$, after which it decreases significantly.

edge (blue) line integrated densities, which increase throughout the discharge in line with the increased fuelling, (e) the electron temperature just inside the pedestal, which decreases slightly over the discharge, and (f) the frequency of Type-I ELMs throughout the discharge. In particular, once the fuelling rate increases strongly after $5 \mathrm{~s}$, the pedestal top temperature decreases noticably. The perturbation coils were switched on between 1.5 and $7.5 \mathrm{~s}$ in the discharge. The Type-I ELMs are mostly mitigated after $4.0 \mathrm{~s}$, as indicated by the ELM frequency trace, with most of the ELMs occurring after this correlated with sawtooth crashes, i.e. a heat pulse from the plasma centre. In the rest of this section, type-I ELMy data are taken from the time window 3.8-4.0 s and in the interval 1-4 ms prior to the ELM crash, while mitigated ELM data are averaged in the time window 4.7-5.0 s. These time ranges were chosen as they feature very different ELM behaviour despite having very similar applied parameters; the gas puff rate is only slightly higher in the ELM mitigated period.

Electron temperature and density profiles from these two phases are shown in figure 10. The type-I ELMy data are shown in red and the mitigated ELM data in blue. A similar pedestal gradient in both the temperature and density profiles exists in both phases. The temperature pedestal top is also similar in both phases, with the only difference being that the pedestal top density is increased in the mitigated phase. This is consistent with previous contributions which have noted a threshold density for the onset of mitigation in the investigated discharges[45, 46]. It is also notable that the 


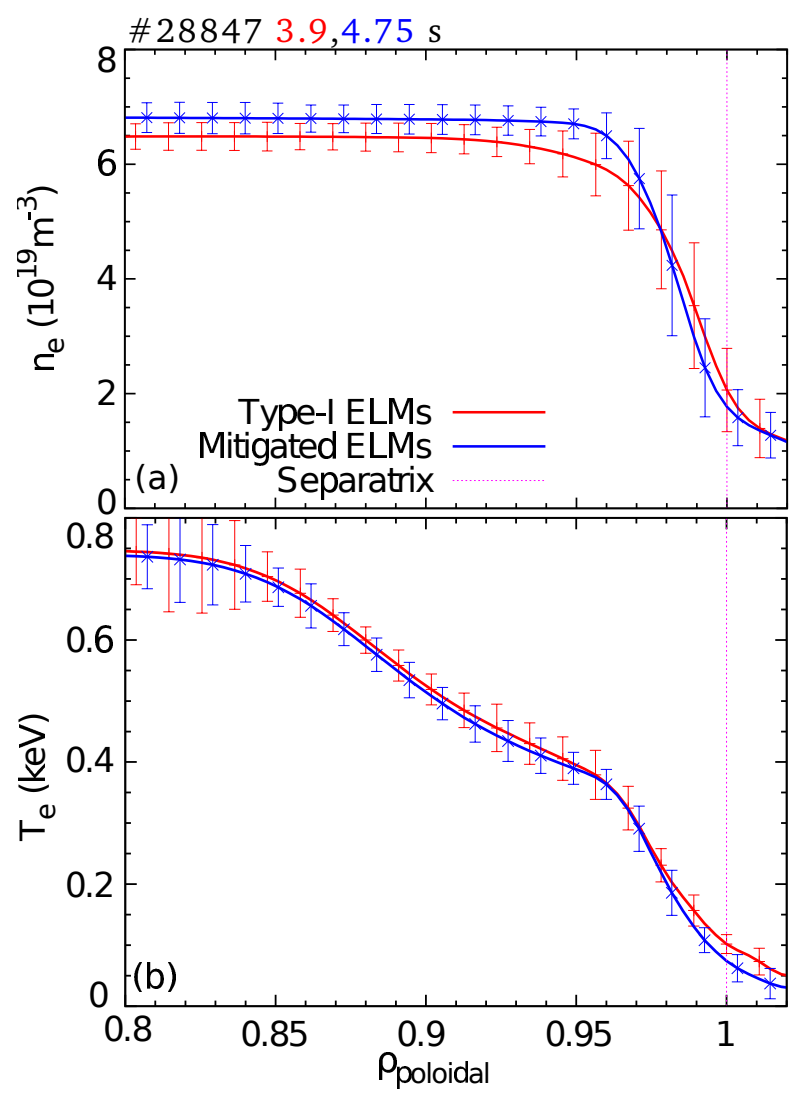

Figure 10. (a) electron density profiles before a type-I ELM (red) and averaged over $300 \mathrm{~ms}$ during the ELM mitigated phase (blue) for discharge \#28847. (b) electron temperature profiles corresponding to the density profiles shown in (a). Notable in both sets of profiles is that there are no significant gradient changes, while the pedestal top density has increased in the ELM-mitigated regime.

measured pedestal width has in this case slightly increased $\left(0.068 \Psi_{\mathrm{N}} \rightarrow 0.078 \Psi_{\mathrm{N}}\right)$, rather than decreased, meaning one of the mechanisms posited by the EPED model for ELM mitigation[16] (that the growth of the pedestal width is interrupted in the middle of the ELM cycle) cannot explain the mitigation in this discharge.

These data were then combined with the magnetic data in the same time windows and used as input for CLISTE. While the real equilibrium in this case is inherently $3 \mathrm{D}$, the $2 \mathrm{D}$ plasma equilibrium corresponding to the toroidal position in which the magnetic sensors are located is used to check for differences in the reconstructed edge current density between the two phases. Since the perturbation coils were switched on during both phases there should be no relative differences, despite the systematic "inaccuracy" in the reconstruction. Indeed, the residuals of the fitted magnetic signals in this discharge are no higher than in a reference companion discharge to the one studied here without magnetic perturbations. The current density calculated by CLISTE is shown in figure 11. The local current density is shown for the two phases in figure 11(a), with the flux surface averaged parallel current density from CLISTE (solid line) and neoclassical theory (dashed line with boxes) shown in figure 11(b). Neither the 


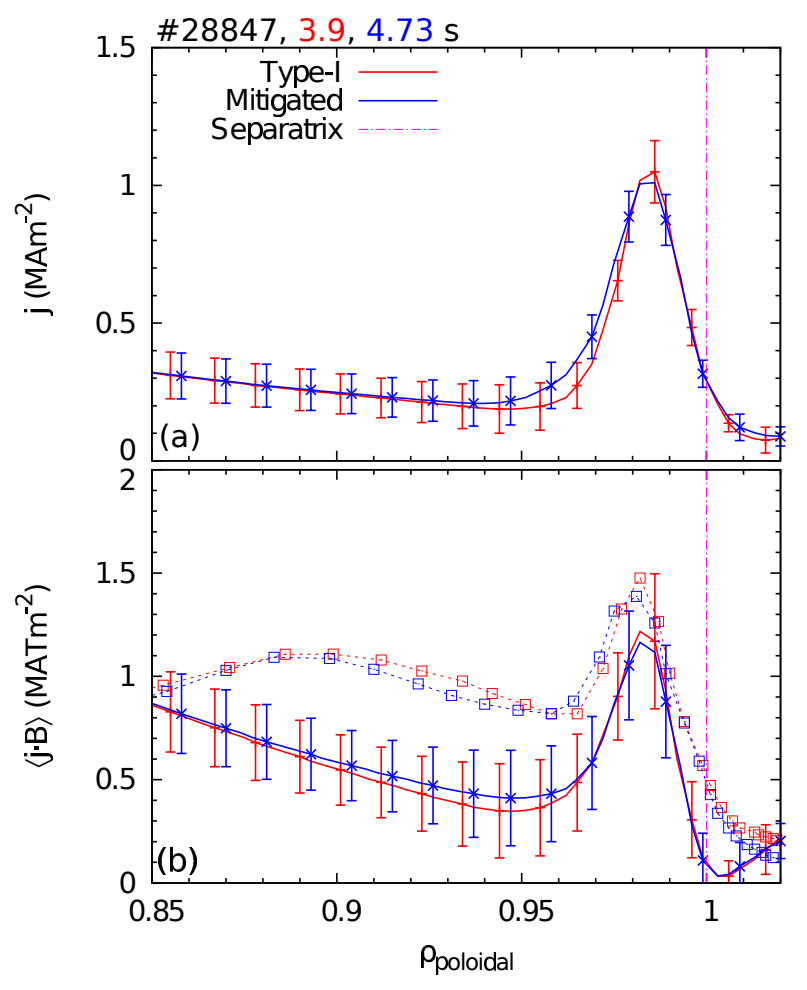

Figure 11. (a) profiles of the local edge current density at the plasma LFS for the type-I ELMy and ELM-mitigated phases of discharge \#28847.(b) $\langle\boldsymbol{j} \cdot \boldsymbol{B}\rangle$ profiles from CLISTE (solid) and neoclassical predictions corresponding to the profiles shown in (a).

magnitude or the shape of either profile change significantly as the plasma moves from a phase with type-I ELMs into a mitigated ELM regime. This is also observed in the calculated neoclassical $\langle\boldsymbol{j} \cdot \boldsymbol{B}\rangle$ profiles. This could be expected as neither the temperature or density profiles change significantly. This indicates that changes in ideal MHD stability are unlikely to be the cause of the small ELMs becoming the dominant ELM type.

\section{5. $\mathrm{N}_{2}$ seeded ELMs}

Tokamak operation with a full metal wall has the advanage of lower chemical sputtering rates while, in the case of tungsten, reducing erosion, or in the case of beryllium, causing an increase of only a low-Z impurity; as such, ITER will be operated with a mixed beryllium wall/tungsten divertor. This wall material mix has been used at JET since 2011[47], while AUG has operated for several years with a full-tungsten wall[48]. While operation with a lower impurity content is possible in both of these machines, a deuterium gas puff is typically required to reduce the occurance of tungsten accumulation in the plasma core. This higher density operation reduces the attainable stored energy in AUG and JET[49, 50, 51]. However, in both devices, confinement was found to recover to levels seen with carbon plasma facing components when nitrogen was puffed into the divertor $[49,51]$. This is shown in figure 12 where a single discharge 
(\#29254) with a reference phase without nitrogen is followed by a nitrogen gas puff. The discharge has a $1 \mathrm{MA}$ plasma current, -2.5 T magnetic field, and average triangularity of 0.29 . While the total heating power remains constant (panel (a)), the plasma stored energy (panel (c)) increases by approximately $40 \%$ once nitrogen is puffed (panel (b)). The electron density remains constant throughout both phases, as shown by the edge and core line intgrated signals in panel $(d)$, while the confinement improvement comes from an increased pedestal top temperature, as seen in panel (e). The ELM frequency is slightly higher during the seeded phase, as shown in panel (f). A timetrace of $\mathrm{Z}_{\text {eff }}$

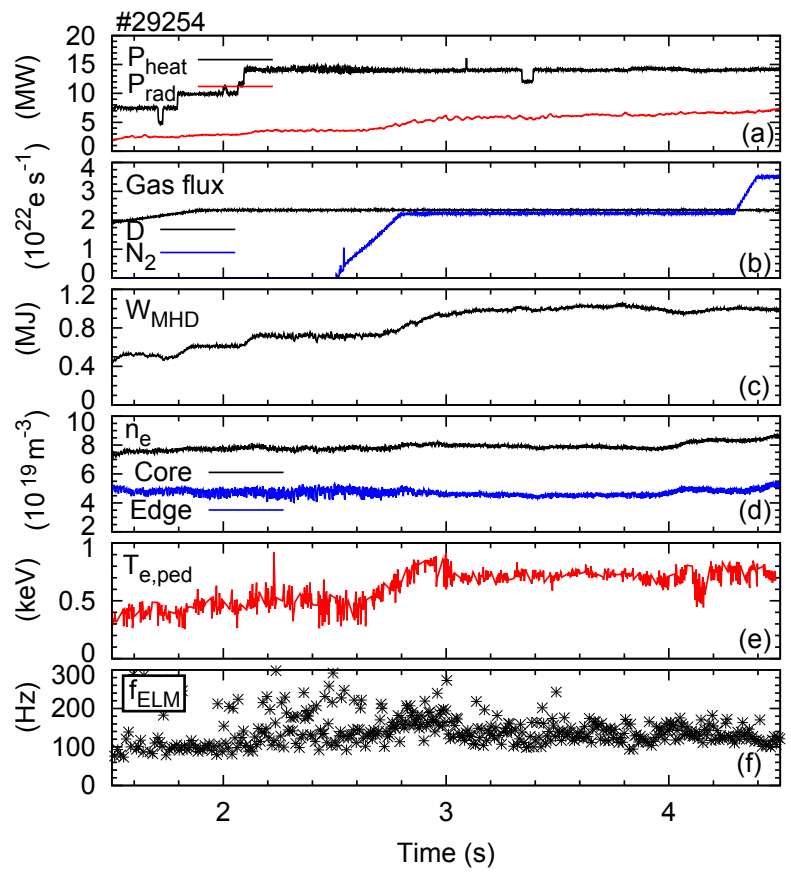

Figure 12. Time traces of (a) total heating power (black), radiated power (red), (b) gas flux from deuterium (black), and nitrogen (blue), (c) plasma stored energy, (d) line integrated core (black) and edge (blue) densities, (e) pedestal top electron temperature and (f) ELM frequency. When the nitrogen puff is applied from $2.5 \mathrm{~s}$, the plasma stored energy, radiated power fraction and pedestal top temperature increase signficantly.

has not been included here, but was shown by Kallenbach et al.[52] to increase from approximately 1.2 to 1.8 when nitrogen is seeded in this discharge.

In addition to improved confinement, ELMs are also observed to be smaller during the nitrogen seeded discharges. This is indicated by the divertor current measurements from discharge \#29254 in figure 13. The absolute magnitude of the rise in the divertor currents is not only smaller in the seeded case, but the time spent at the peak value is also shorter. This longer ELM crash in the reference case corresponds to the observed "second ELM phase" postulated by Schneider et al.[53]. The cause of this second phase is currently unknown, but is almost always observed in AUG discharges without impurity seeding and has also been observed in JET since the installation of the ITERlike wall[50]. In the contribution from Schneider et al., it was shown that the ELMs in 
a nitrogen seeded discharge consisted only of a drop at the pedestal top. The ELMs in a companion reference discharge consisted of a drop at the pedestal top and also a collapse of the steep gradient region which occurred during this flattop phase indicated in the divertor current measurements. While an analysis of the edge current density was presented in [53], a further more detailed analysis will be presented here in order to compare this scenario to other ELM mitigation methods.

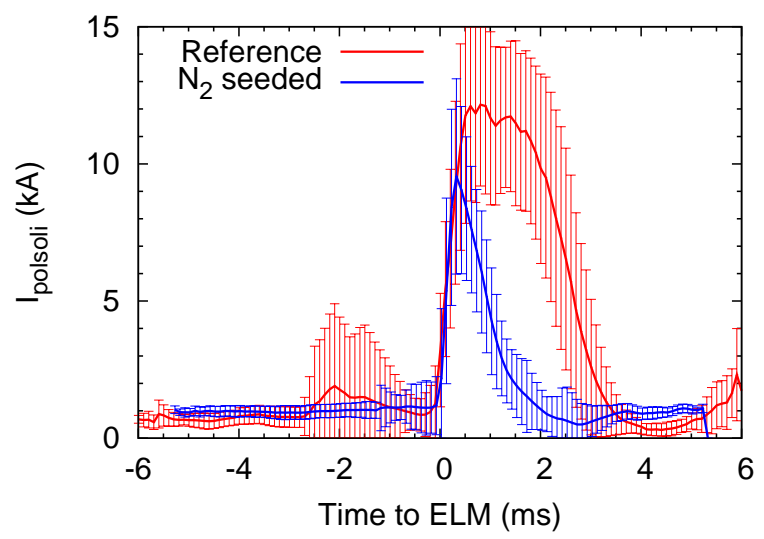

Figure 13. ELM synchronised outer divertor currents for discharge \#29254 showing the striking difference in both the peak magnitude and duration of this peak between the reference phase (red) and the seeded phase (blue). The error bars indicate the standard deviation of the currents in $0.1 \mathrm{~ms}$ time windows relative to the ELM crash. Onset of recovery from the crash, as well as the total ELM cycle, is significantly faster in the seeded case.

The pre-ELM pedestal profile changes are shown for discharge \#29254 as solid lines in figure 14 for (a): electron density and (b): electron temperature. Data for the reference phase (blue) were taken between 1.8 and $2.3 \mathrm{~s}$ in the discharge, while the data for the seeded phase (red) were taken between 3 and $4 \mathrm{~s}$. Here, pre-ELM data are taken from $2 \mathrm{~ms}$ before the ELM onset until the onset time, as indicated by the divertor current measurements, while the post-ELM time is taken as 0-2 ms. Figure 14(a) shows the electron density profiles, which show no change as nitrogen seeding is applied. Figure 14(b) shows the increase of the electron temperature pedestal top, which, when combined with the stiff core $\mathrm{T}_{\mathrm{e}}$ profiles, causes the increase in the plasma stored energy. The smaller ELM size in the nitrogen seeded phase (35 kJ losses in the reference phase vs. $20 \mathrm{~kJ}$ in the seeded phase) is also clearly indicated in figure 14, where the dashed lines show the post-ELM profiles. In the case of the density, the reference phase exhibits both a drop of the pedestal top value as well as the gradient, while in the seeded phase only the pedestal top value is affected. This is seen even more prominently in the temperature profile, where the reference profile flattens completely while there is only a small drop in the pedestal top value in the seeded phase, consistent with the observations by Schneider et al.[53].

In order to compare the edge current density in both of these phases, the data shown in figure 14 were used as input for CLISTE with the assumption $\mathrm{T}_{\mathrm{i}}=\mathrm{T}_{\mathrm{e}}$. Magnetic data 


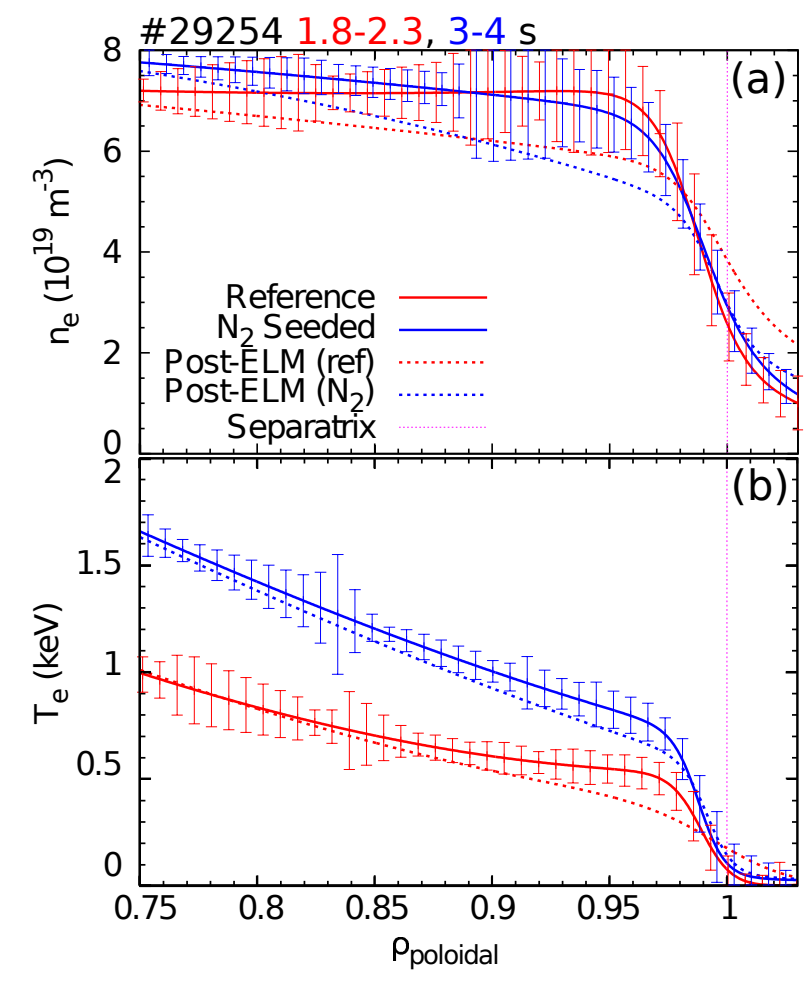

Figure 14. Profiles of (a): electron density and (b): electron temperature for the reference (red) and $\mathrm{N}_{2}$ seeded (blue) phases of discharge \#29254. Shown are the preELM (solid) and post-ELM (dashed) profiles for each phase, indicating the difference in the behaviour of the pedestal gradients between the two ELM types.

were also taken in the same ELM averaged time windows. The local current density profiles at the LFS midplane from the CLISTE fits are shown in figure 15. The solid lines show the pre-ELM profiles while the dashed lines show the post-ELM profiles. The pre-ELM profiles are similar in shape, though the edge current density peak is higher in the seeded case, corresponding to the increased pressure gradient.

Much more striking is the difference in the current density profiles just after the ELM crash. In the reference case, the edge current density profile has completely flattened, as is typical of a type-I ELM. However, in the seeded case, there is still a strong edge peak indicating that the plasma has not completely relaxed the free energy available to it for some reason, or that the hypothesised second phase of the crash is prohibited in the nitrogen seeded case. To put this in context with the data shown in figure 4; it appears that applying gas puff to an otherwise similar discharge scenario decreases the ELM size, but increases the pressure gradient and current density collapse after the ELM crash. This gradient collapse can then be mitigated by nitrogen seeding. It is not clear what effect nitrogen seeding would have in the absense of a gas puff; a dedicated experiment for this scenario is required. The parallel current density is shown in figure 16, where the CLISTE $\langle\boldsymbol{j} \cdot \boldsymbol{B}\rangle$ (solid lines) is plotted with the neoclassical predictions using the $\mathrm{Z}_{\text {eff }}$ values given by a Bayesian Bremsstrahlung and charge exchange analysis[54] (square points and dashed lines). Figure 16(a) shows 


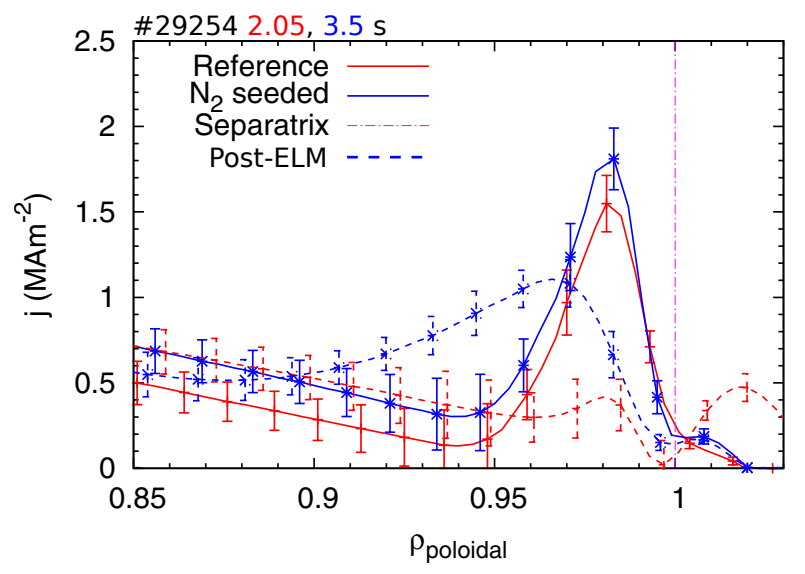

Figure 15. Edge current density for (a): pre- and (b): post-ELM timepoints in the reference (red) and seeded discharge (blue). The pre-ELM profiles have a similar basic shape, with the seeded case having a higher peak which is closer to the separatrix while the post-ELM profiles show large differences, reflecting the differences in the crash sizes.

the pre-ELM values, where the parallel current density is the same in the two cases. Typically, an increased pedestal top temperature or pedestal pressure gradient indicate that the bootstrap current increases. This lack of change in the profiles could have important implications for the stabilisation of different modes. A strongly flattened (or reversed) local magnetic shear, caused by the strong local edge current density peak could stabilise ballooning modes, while the peeling mode is stabilised by the higher pressure gradient while the drive stays the same.

Regarding the post-ELM profiles, the reference case shows a complete loss of the edge current density peak, while peaking strongly in the SOL; this corresponds to the large divertor currents in the reference case shown in figure 13. There is still a clear edge current density peak in the seeded case, similar to the findings for the local current density, which also compares well to the neoclassical prediction. Again, the higher measured $\mathrm{Z}_{\text {eff }}$ was necessary to obtain good agreement between CLISTE and neoclassics, implying that the impurity content in the pedestal is not decreased substantially by the ELM crash. For the reference case, however, the same quality of agreement cannot be found. The neoclassical prediction also demonstrates the flattening of the edge current density profile, but to a lesser extent than observed with CLISTE. In the two cases presented here, the ELM induced toroidal electric field was not included since only single timepoints relative to the ELM were taken. Instead, the toroidal loop voltage measured outside the plasma was used, which is not an accurate indication of the parallel electric field profile in the plasma shortly after an ELM crash[17, 23].

The competition between the increased $\mathrm{Z}_{\text {eff }}$, which increases the collisionality, and the increased electron temperature, which both decreases the collisionality and provides a larger drive for the bootstrap current, is shown more clearly in figure 17 for three discharges with nitrogen seeded and reference counterparts. In this figure, the edge current density from CLISTE (stars) is plotted as a function of the pedestal top 


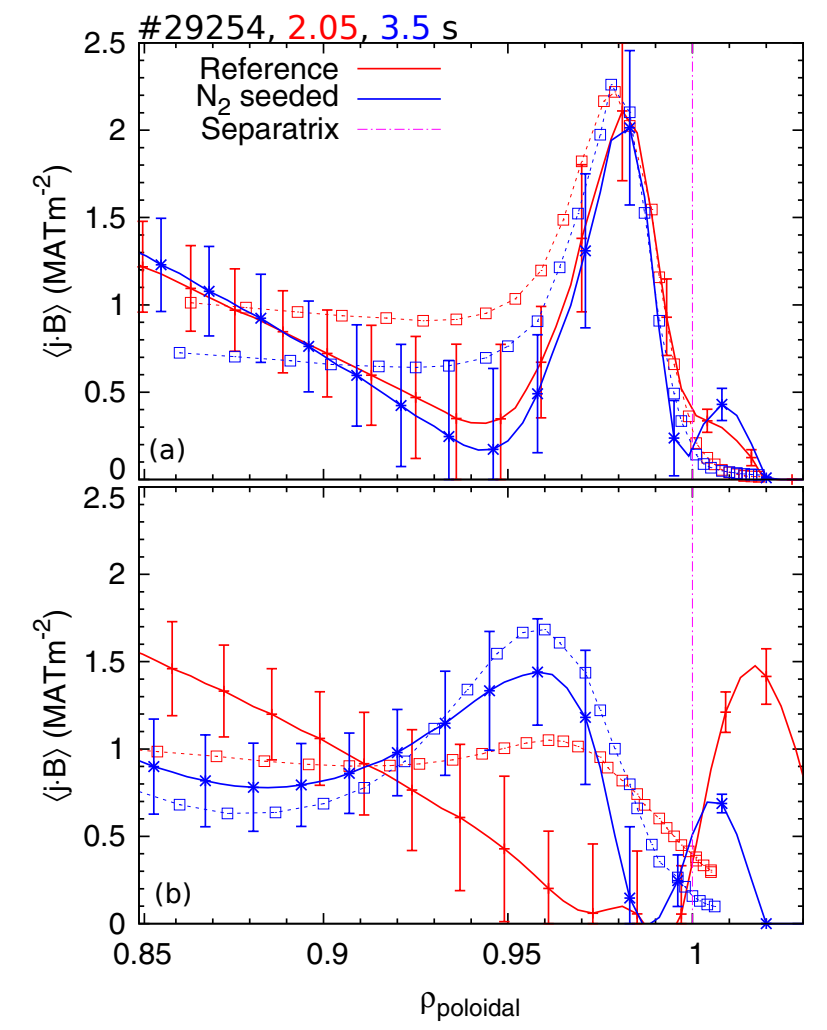

Figure 16. Profiles of the flux surface averaged current density for (a):pre- and (b)-post ELM profiles in the reference (red) and seeded (blue) discharges. The solid lines show the CLISTE results while the dashed lines with boxes show the neoclassical prediction. This figure shows the striking finding that the flux surface averaged current density is the same in the nitrogen seeded discharge as it is in the reference case, contrasting with the larger peak found for the local LFS current density.

temperature, showing the change of the edge current density as the confinement increases (red indicates the reference phases, blue the seeded). The boxes show the neoclassical current density calculated with the measured value of $\mathrm{Z}_{\text {eff }}$, while the blue circles show the calculated current density using the value of $\mathrm{Z}_{\text {eff }}$ before nitrogen is added. While for lower pedestal top temperatures the change effected by the impurity seeding is not so strong, at higher temperatures the neoclassical current density remains close to the reference value despite the edge collisionality dropping and the electron temperature gradient, one of the drives for the bootstrap current, increasing. The three cases shown in figure 17 show the three different resulting effects on the edge current density: a decrease for the \#24681/2 pair; no change in discharge \#29524; and an increase for discharge \#29874. Which one of these scenarios occurs depends on the initial plasma beta as well as the amount of nitrogen which is seeded. The mechanism behind increased plasma confinement with impurity seeding is still not understood and, thus, no firm statement on why a particular scenario takes place for a given starting plasma can be made at the moment. These observations indicate that a reduced current density could allow access to higher pressure gradients. The role of the reduced current density in the mitigation of 


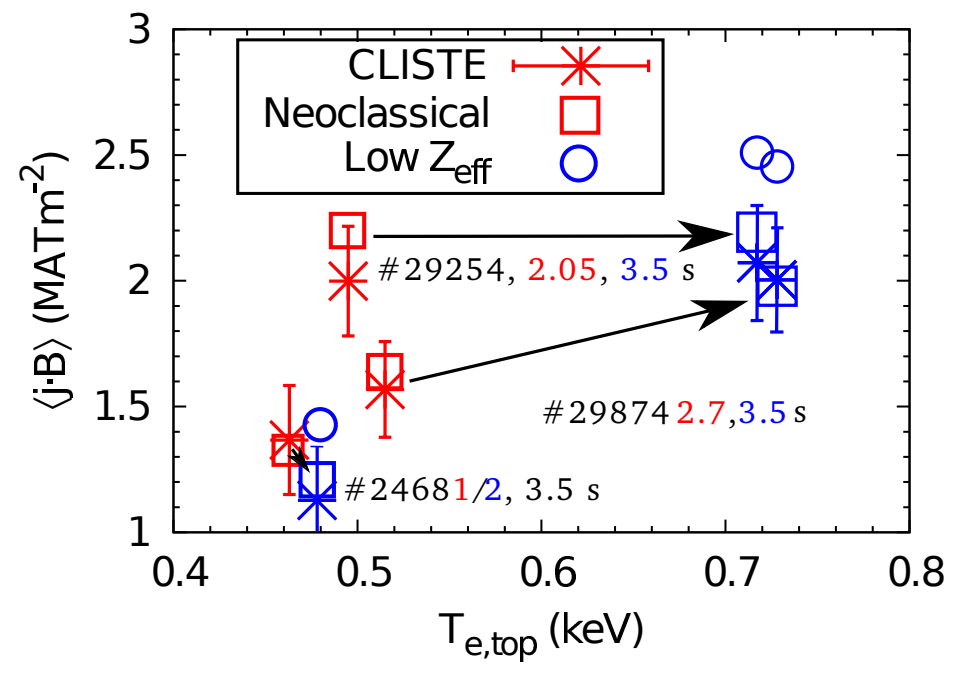

Figure 17. Flux surface averaged parallel edge current density with and without nitrogen seeding as a function of pedestal top temperature. The red symbols indicate the reference points from three discharges, the blue the nitrogen seeded counterparts. The stars show the current density from CLISTE with $1 \sigma$ confidence intervals, while the boxes indicate the calculated neoclassical current denstiy using the measured $\mathrm{Z}_{\text {eff }}$ values. The circles indicate the current density calculated using the improved temperature values, but the unseeded value of $Z_{\text {eff }}$.

the second ELM phase is unclear; all three of these discharge pairs feature reduced ELM lengths in the nitrogen seeded phases, with the common changes being a reduced current density with respect to the pressure gradient, a lower pedestal top collisionality, and a lower SOL temperature. Since lower pedestal top collisionality is typically correlated with increased ELM size[38], this is unlikely to be the cause. This means that the ratio of edge current density to pressure gradient and the SOL temperature are the possible factors in determining the ELM length.

To summarise, the application of nitrogen seeding in AUG has been found to both improve total confinement and to reduce the size of type-I ELMs. In line with the increased pressure gradient, a higher LFS edge current density peak is observed when $\mathrm{N}_{2}$ seeding is applied, while the flux surface averaged current density remains the same or may even decrease. The higher $\mathrm{Z}_{\mathrm{eff}}$ in the seeded cases was shown to have a dramatic impact on the form of the average parallel current density. The post-ELM profiles of the flux surface averaged current density echoed those of the local current density, with the seeded case retaining a significant peak. Excellent agreement between the neoclassical prediction and CLISTE in the pre-ELM phases of both timepoints as well as in the post-ELM phase of the nitrogen seeded timepoint is observed. This could imply that the losses in the seeded case proceed according to neoclassical transport while typical type-I ELMs experience a more rapid loss mechanism, or that the ions have a different temporal behaviour in both cases. 


\section{Conclusions}

This paper has shown several examples of ELMs mitigated via an increased gas puff. Type-I ELMs in an otherwise similar high performance H-mode are smaller when a gas puff is applied. Despite the large difference in pedestal top collisionality, the resulting current denstiy in the pedestal is almost unchanged, indicating that it is the total pressure profile shape and magnitude which has the larger impact on the resuling current density, rather than the individual temperature and density contributions. The only significant difference noted was a decreased current density at the separatrix when gas fuelling was applied. However, when external gas puffing is combined with a change in the plasma configuration from lower single null to an almost double null shape, the characteristics of both the pressure profile and the edge current density are changed dramatically; both the pressure gradient and edge current density decrease significantly in the Type-II ELMing regime. However, due to the results from section 2 it would appear that the lower edge current density is not simply a result of an increased density; the pressure gradient needs to decrease first in order for the current density to decrease. While a lower current density may be a requirement for entry into the ballooning domianted small ELM regime, it is not likely to be the trigger for it. This is further demonstrated in the case of ELM mitigation by external magnetic perturbations, where neither the pressure profile or the edge current density change when moving from a regime featuring a mixture of Type-I and small ELMs to a regime with only small ELMs. The nature of this evolution is unknown and cannot be described by either changes to the main parameters which determine linear peeling-ballooning stability. This may point to a threshold effect, the study of which is more suited to database methods of analysis.

The small ELMs which occur in nitrogen seeded plasmas appear to consist of only one part of a typical two part ELM crash. This two part ELM crash[53] has been observed to consist of an initial crash at the pedestal top, followed by a secondary crash of the pedestal gradients. The reconstructed current density in nitrogen seeded plasmas exhibits behaviour that is not seen in any of the other ELM mitigation scenarios, where the local and flux surface averaged current density profiles varied in a similar fashion. In this case, the local edge current density increases due to an increased Pfirsch-Schlüter drive. However, despite the increased pedestal gradients, the flux surface averaged parallel current density reacts in a different fashion depending on the magnitude of the increase of the temperature gradient, which acts to increase the current density, and the increase of the effective charge in the pedestal, which acts to decrease it. These two effects compete against each other, resulting in three possible scenarios: an increased $\langle\boldsymbol{j} \cdot \boldsymbol{B}\rangle$, an unchanged $\langle\boldsymbol{j} \cdot \boldsymbol{B}\rangle$, or a decreased $\langle\boldsymbol{j} \cdot \boldsymbol{B}\rangle$. All of these possibilities have been observed and are shown in figure 17; which scenario results depends on the amount of nitrogen seeding which is applied and the plasma beta before seeding takes place. What is clear is that in all cases, the resulting $\langle\boldsymbol{j} \cdot \boldsymbol{B}\rangle$, which drives the peeling mode, has increased less than would be predicted by an increased pressure gradient. This could 
mean that there is less drive for the peeling mode relative to the ballooning mode, causing the more benign ELMs observed during nitrogen seeded plasmas. Additionally, the increased local current density could alter the local shear profile, making the plasma more stable against ballooning modes. This latter effect may also be responsible for the increased confinement. However, both of these effects need to be checked with detailed MHD stability analysis to verify this hypothesis. An implication of the increased $\mathrm{Z}_{\text {eff }}$ and its subsequent impact on the edge current density being ultimately responsible for the confinement improvement and smaller ELMs is that the specific impurity used should not matter. Experiments are planned for the upcoming AUG experimental campaign to verify if this is indeed the case or if there are other effects which must be taken into account.

With respect to operation with mitigated ELMs in ITER, operation with type-II ELMs is not likely to be possible due to the strong shaping and high collisionality requirements. ELM mitigation with magnetic perturbations has so far only been demonstrated at ITER relevant collisionalities at the DIII-D tokamak[43], though this was at low density. The question of operation at high confinement when these perturbations are present is still open, however. If the choice of impurity impurity species does not otherwise degrade perfromance, then extrinsic impurity seeding is a promising operational scenario for ITER as it offers improved confinement with smaller ELMs over a non-seeded scenario. This regime also has benefits for reduction of the heat load on the divertor during inter-ELM periods in next generation tokamak devices.

\section{References}

[1] G. Federici, C.H. Skinner, J.N. Brooks, J.P. Coad, C. Grisolia, A.A. Haasz, V. Philipps, C.S. Pitcher, J. Roth, W.R. Wampler, and D.G. Whyte. Plasma material interactions in current tokamaks and their implications for next step fusion reactors. Nuclear Fusion, 41(12R), 2001.

[2] Y. Kamada, R. Yoshino, Y. Neyatani, M. Sato, S. Tokuda, M. Azumi, S. Takeji, K. Ushigusa, T. Fukuda, M. Mori, T. Takizuka, and JT-60U Team. Onset condition for ELMs in JT-60U. Plasma Physics and Controlled Fusion, 38:1387-1391, 1996.

[3] J. Stober, M. Maraschek, G.D. Conway, O. Gruber, A. Herrmann, A.C.C. Sips, W. Treutterer, H. Zohm, and Asdex Upgrade Team. Type II ELMy H modes on ASDEX Upgrade with good confinement at high density. Nuclear Fusion, 1123, 2001.

[4] A. Kirk, H.W. Muller, E. Wolfrum, H. Meyer, A. Herrmann, T. Lunt, V. Rohde, and P. Tamain. Comparison of small edge-localized modes on MAST and ASDEX Upgrade. Plasma Physics and Controlled Fusion, 53(9):095008, September 2011.

[5] P.T. Lang, a. Burckhart, M. Bernert, L. Casali, R. Fischer, O. Kardaun, G. Kocsis, M. Maraschek, a. Mlynek, B. Plöckl, M. Reich, F. Ryter, J. Schweinzer, B. Sieglin, W. Suttrop, T. Szepesi, G. Tardini, E. Wolfrum, D. Zasche, and H. Zohm. ELM pacing and high-density operation using pellet injection in the ASDEX Upgrade all-metal-wall tokamak. Nuclear Fusion, 54(8):083009, August 2014.

[6] L.R. Baylor, T.C. Jernigan, P.B. Parks, G. Antar, N.H. Brooks, S.K. Combs, D.T. Fehling, C.R. Foust, W.a. Houlberg, and G.L. Schmidt. Comparison of deuterium pellet injection from different locations on the DIII-D tokamak. Nuclear Fusion, 47(11):1598-1606, November 2007.

[7] A.W. Degeling, Y.R. Martin, J.B. Lister, L. Villard, V.N. Dokouka, V.E. Lukash, and R.R. 
Impact of $T_{\mathrm{e}}$ and $n_{\mathrm{e}}$ on edge current density profiles

Khayrutdinov. Magnetic triggering of ELMs in TCV. Plasma Physics and Controlled Fusion, 45:1637-1655, 2003.

[8] S.P. Gerhardt, J-W. Ahn, J.M. Canik, R. Maingi, R. Bell, D. Gates, R. Goldston, R. Hawryluk, B.P. Le Blanc, J. Menard, a.C. Sontag, S. Sabbagh, and K. Tritz. First observation of ELM pacing with vertical jogs in a spherical torus. Nuclear Fusion, 50(6):064015, June 2010.

[9] E. de la Luna. The effect of ELM pacing by vertical kicks on the access to stationary H-mode with $\mathrm{H}=1$. In Int. Conf. on Fusion Energy, San Diego, CA, 2012.

[10] W.W. Xiao, P.H. Diamond, X.L. Zou, J.Q. Dong, X.T. Ding, L.H. Yao, B.B. Feng, C.Y. Chen, W.L. Zhong, M. Xu, B.S. Yuan, T. Rhee, J.M. Kwon, Z.B. Shi, J. Rao, G.J. Lei, J.Y. Cao, J. Zhou, M. Huang, D.L. Yu, Y. Huang, K.J. Zhao, Z.Y. Cui, X.M. Song, Y.D. Gao, Y.P. Zhang, J. Cheng, X.Y. Han, Y. Zhou, Y.B. Dong, X.Q. Ji, Q.W. Yang, Yi Liu, L.W. Yan, X.R. Duan, and Yong Liu. ELM mitigation by supersonic molecular beam injection into the H-mode pedestal in the HL-2A tokamak. Nuclear Fusion, 52(11):114027, November 2012.

[11] W.W. Xiao, P.H. Diamond, W.C. Kim, L.H. Yao, S.W. Yoon, X.T. Ding, S.H. Hahn, J. Kim, M. Xu, C.Y. Chen, B.B. Feng, J. Cheng, W.L. Zhong, Z.B. Shi, M. Jiang, X.Y. Han, Y.U. Nam, W.H. Ko, S.G. Lee, J.G. Bak, J.W. Ahn, H.K. Kim, H.T. Kim, K.P. Kim, X.L. Zou, S.D. Song, J.I. Song, Y.W. Yu, T. Rhee, J.M. Kwon, X.L. Huang, D.L. Yu, K.D. Lee, S.I. Park, M. Jung, S. Zoletnik, M. Lampert, G.R. Tynan, Y.S. Bae, J.G. Kwak, L.W. Yan, X.R. Duan, Y.K. Oh, and J.Q. Dong. ELM mitigation by supersonic molecular beam injection: KSTAR and HL-2A experiments and theory. Nuclear Fusion, 54(2):023003, February 2014.

[12] T. Evans, R. Moyer, P. Thomas, J. Watkins, T. Osborne, J. Boedo, E. Doyle, M. Fenstermacher, K. Finken, R. Groebner, M. Groth, J. Harris, R. La Haye, C. Lasnier, S. Masuzaki, N. Ohyabu, D. Pretty, T. Rhodes, H. Reimerdes, D. Rudakov, M. Schaffer, G. Wang, and L. Zeng. Suppression of Large Edge-Localized Modes in High-Confinement DIII-D Plasmas with a Stochastic Magnetic Boundary. Physical Review Letters, 92(23):235003, June 2004.

[13] Y. Liang, H. Koslowski, P. Thomas, E. Nardon, B. Alper, P. Andrew, Y. Andrew, G. Arnoux, Y. Baranov, M. Bécoulet, M. Beurskens, T. Biewer, M. Bigi, K. Crombe, E. De La Luna, P. de Vries, W. Fundamenski, S. Gerasimov, C. Giroud, M. Gryaznevich, N. Hawkes, S. Hotchin, D. Howell, S. Jachmich, V. Kiptily, L. Moreira, V. Parail, S. Pinches, E. Rachlew, and O. Zimmermann. Active Control of Type-I Edge-Localized Modes with $\mathrm{n}=1$ Perturbation Fields in the JET Tokamak. Physical Review Letters, 98(26):265004, June 2007.

[14] W. Suttrop, A. Herrmann, A. Kallenbach, P. T Lang, T. Lunt, T. Eich, J. C Fuchs, S Gu, S. Günter, M. Maraschek, R. McDermott, A. Mlynek, T. Pütterich, M. Rott, T. Vierle, E. Wolfrum, Q. Yu, I. Zammuto, and H. Zohm. First Observation of Edge Localized Modes Mitigation with Resonant and Nonresonant Magnetic Perturbations in ASDEX Upgrade. Physical Review Letters, 106(22):1-4, June 2011.

[15] H.R. Wilson, S.C. Cowley, A. Kirk, and P.B. Snyder. Magneto-hydrodynamic stability of the $\mathrm{H}$-mode transport barrier as a model for edge localized modes: an overview. Plasma Physics and Controlled Fusion, 48(5A):A71-A84, May 2006.

[16] P. B. Snyder, T. H. Osborne, K. H. Burrell, R. J. Groebner, a. W. Leonard, R. Nazikian, D. M. Orlov, O. Schmitz, M. R. Wade, and H. R. Wilson. The EPED pedestal model and edge localized mode-suppressed regimes: Studies of quiescent H-mode and development of a model for edge localized mode suppression via resonant magnetic perturbations. Physics of Plasmas, 19(5):056115, 2012.

[17] A. Burckhart, E. Wolfrum, R. Fischer, K. Lackner, and H. Zohm. Inter-ELM behaviour of the electron density and temperature pedestal in ASDEX Upgrade. Plasma Physics and Controlled Fusion, 52(10):105010, October 2010.

[18] D Dickinson, S Saarelma, R Scannell, a Kirk, C M Roach, and H R Wilson. Towards the construction of a model to describe the inter-ELM evolution of the pedestal on MAST. Plasma Physics and Controlled Fusion, 53(11):115010, November 2011.

[19] P. Maget, J.-F. Artaud, M. Bécoulet, T. Casper, J. Faustin, J. Garcia, G.T.a. Huijsmans, a. Loarte, 
and G. Saibene. MHD stability of the pedestal in ITER scenarios. Nuclear Fusion, 53(9):093011, September 2013.

[20] G T a Huysmans, Home Search, Collections Journals, About Contact, My Iopscience, Plasma Phys, and I P Address. External kink (peeling) modes in x-point geometry. Plasma Physics and Controlled Fusion, 47(12):2107-2121, December 2005.

[21] D. Thomas, a. Leonard, L. Lao, T. Osborne, H. Mueller, and D. Finkenthal. Measurement of Pressure-Gradient-Driven Currents in Tokamak Edge Plasmas. Physical Review Letters, 93(6):065003, August 2004.

[22] M.F.M. De Bock, J. Citrin, S. Saarelma, D. Temple, N.J. Conway, A. Kirk, H. Meyer, and C.A. Michael. Measurements of the edge current evolution and comparison with neoclassical calculations during MAST H-modes using motional Stark effect. Plasma Physics and Controlled Fusion, 54(2):025001, February 2012.

[23] M.G. Dunne, P.J. McCarthy, E. Wolfrum, R. Fischer, L. Giannone, and a. Burckhart. Measurement of neoclassically predicted edge current density at ASDEX Upgrade. Nuclear Fusion, 52(12):123014, December 2012.

[24] O. Sauter, C. Angioni, and Y. R. Lin-Liu. Neoclassical conductivity and bootstrap current formulas for general axisymmetric equilibria and arbitrary collisionality regime. Physics of Plasmas, 6(7):2834, 1999.

[25] O. Sauter, C. Angioni, and Y. R. Lin-Liu. Erratum: Neoclassical conductivity and bootstrap current formulas for general axisymmetric equilibria and arbitrary collisionality regime [Phys. Plasmas 6, 2834 (1999)]. Physics of Plasmas, 9(12):5140, 2002.

[26] P.J. McCarthy. Analytical solutions to the GradShafranov equation for tokamak equilibrium with dissimilar source functions. Physics of Plasmas, 6(9):3554, 1999.

[27] P.J. McCarthy. Identification of edge-localized moments of the current density profile in a tokamak equilibrium from external magnetic measurements. Plasma Physics and Controlled Fusion, 54(1):015010, January 2012.

[28] Philip Adrian Schneider. Characterization and scaling of the tokamak edge transport barrier. PhD thesis, LMU, 2012.

[29] N.A. Salmon. First electron temperature edge measurements on the ASDEX Upgrade tokamak using a heterodyne radiometer. International Journal of Infrared and Millimeter Waves, 15(1):53-60, 1994.

[30] H. Murmann, S. Gotsch, H. Rohr, H. Salzmann, and K. H. Steuer. The Thomson scattering systems of the ASDEX upgrade tokamak. Review of Scientific Instruments, 63(10):4941, 1992.

[31] B. Kurzan and H.D. Murmann. Edge and core Thomson scattering systems and their calibration on the ASDEX Upgrade tokamak. The Review of scientific instruments, 82(10):103501, October 2011.

[32] E. Wolfrum, F. Aumayr, D. Wutte, Hp. Winter, E. Hintz, D. Rusbuldt, and R. P. Schorn. Fast lithium-beam spectroscopy of tokamak edge plasmas. Review of Scientific Instruments, 64(8):2285, 1993.

[33] R. Fischer, E. Wolfrum, and J. Schweinzer. Probabilistic lithium beam data analysis. Plasma Physics and Controlled Fusion, 50(8):085009, August 2008.

[34] O. Gehre. The HCN-laser-interferometer of the divertor tokamak ASDEX. International Journal of Infrared and Millimeter Waves, 5(3):369-379, March 1984.

[35] P.A. Schneider, E. Wolfrum, R.J. Groebner, T.H. Osborne, M.N.A. Beurskens, M.G. Dunne, B. Kurzan, T. Pütterich, and E. Viezzer. Analysis of temperature and density pedestal gradients in AUG, DIII-D and JET. Nuclear Fusion, 53(7):073039, July 2013.

[36] A. Kallenbach, A. Carlson, G. Pautasso, A. Peeters, U. Seidel, and H.-P. Zehrfeld. Electric currents in the scrape-off layer in ASDEX Upgrade. Journal of Nuclear Materials, 290-293:639-643, March 2001.

[37] R.A. Pitts, S. Alberti, P. Blanchard, J. Horacek, H. Reimerdes, and P.C. Stangeby. ELM driven divertor target currents on TCV. Nuclear Fusion, 43(10):1145-1166, October 2003. 
[38] A. Loarte, G. Saibene, R. Sartori, D. Campbell, M. Becoulet, L.D. Horton, T. Eich, A. Herrmann, G.F. Matthews, N. Asakura, A. Chankin, A. Leonard, G. Porter, G. Federici, G. Janeschitz, M. Shimada, and M. Sugihara. Characteristics of type I ELM energy and particle losses in existing devices and their Characteristics of type I ELM energy and particle. Plasma Physics and Controlled Fusion, 45:1549-1569, 2003.

[39] J.E. Boom, E. Wolfrum, I.G.J. Classen, P.C. de Vries, M. Maraschek, W. Suttrop, C.P. Pérez von Thun, a.J.H. Donné, B.J. Tobias, C.W. Domier, N.C. Luhmann, and H.K. Park. Characterization of broadband MHD fluctuations during type-II edge localized modes as measured in 2D with ECE-imaging at ASDEX Upgrade. Nuclear Fusion, 52(11):114004, November 2012.

[40] E. Wolfrum, M. Bernert, J.E. Boom, A. Burckhart, I.G.J. Classen, G.D. Conway, T. Eich, R. Fischer, A. Gude, A. Herrmann, N.C. Luhmann, M. Maraschek, R. McDermott, H.K. Park, T. Pütterich, J. Vicente, B. Wieland, and M. Willensdorfer. Characterization of edge profiles and fluctuations in discharges with type-II and nitrogen-mitigated edge localized modes in ASDEX Upgrade. Plasma Physics and Controlled Fusion, 53(8):085026, August 2011.

[41] S. Saarelma, A. Alfier, M.N.A. Beurskens, R. Coelho, H.R. Koslowski, Y. Liang, and I. Nunes. MHD stability analysis of small ELM regimes in JET. Plasma Physics and Controlled Fusion, 51(3):035001, March 2009.

[42] A. Kirk, E. Nardon, P. Tamain, P. Denner, H. Meyer, S. Mordijck, and D. Temple. The effect of resonant magnetic perturbations on L and H-mode plasmas on MAST. Journal of Nuclear Materials, 415(1):S910-S913, August 2011.

[43] T.E. Evans, R.A Moyer, J.G. Watkins, T.H. Osborne, P.R. Thomas, M. Becoulet, J.A. Boedo, E.J. Doyle, M.E. Fenstermacher, K.H. Finken, R.J. Groebner, M. Groth, J.H. Harris, G.L. Jackson, R.J. La Haye, C.J. Lasnier, S. Masuzaki, N. Ohyabu, D.G. Pretty, H. Reimerdes, T.L. Rhodes, D.L. Rudakov, M.J. Schaffer, M.R. Wade, G. Wang, W.P. West, and L. Zeng. Suppression of large edge localized modes with edge resonant magnetic fields in high confinement DIII-D plasmas. Nuclear Fusion, 45(7):595-607, July 2005.

[44] W. Suttrop, L. Barrera Orte, T. Eich, R. Fischer, J.C. Fuchs, L. Giannone, M. Ko, and P.T. Lang. Mitigation of Edge Localised Modes with small non-axisymmetric magnetic perturbations in ASDEX Upgrade. In IAEA, pages 1-8, 2012.

[45] W. Suttrop, L. Barrera, A. Herrmann, R.M. McDermott, T. Eich, R. Fischer, B. Kurzan, P.T. Lang, A. Mlynek, T. Pütterich, S.K. Rathgeber, M. Rott, T. Vierle, E. Viezzer, M. Willensdorfer, E. Wolfrum, and I. Zammuto. Studies of edge localized mode mitigation with new active invessel saddle coils in ASDEX Upgrade. Plasma Physics and Controlled Fusion, 53(12):124014, December 2011.

[46] R. Fischer, J.C. Fuchs, R. McDermott, S.K. Rathgeber, W. Suttrop, M. Willensdorfer, and E. Wolfrum. Spatiotemporal response of plasma edge density and temperature to nonaxisymmetric magnetic perturbations at ASDEX Upgrade. Plasma Physics and Controlled Fusion, 54(11):115008, November 2012.

[47] L. Horton. The JET ITER-like wall experiment: First results and lessons for ITER. Fusion Engineering and Design, 88(6-8):434-439, October 2013.

[48] A. Kallenbach, R. Dux, M. Mayer, R. Neu, T. Pütterich, V. Bobkov, J.C. Fuchs, T. Eich, L. Giannone, O. Gruber, A. Herrmann, L.D. Horton, C.F. Maggi, H. Meister, H.W. Müller, V. Rohde, A. Sips, A. Stäbler, and J. Stober. Non-boronized compared with boronized operation of ASDEX Upgrade with full-tungsten plasma facing components. Nuclear Fusion, 49(4):045007, April 2009.

[49] J. Schweinzer, A.C.C. Sips, G. Tardini, P.A. Schneider, R. Fischer, J.C. Fuchs, O. Gruber, J. Hobirk, A. Kallenbach, R.M. McDermott, R. Neu, T. Pütterich, S.K. Rathgeber, J. Stober, and J. Vicente. Confinement of improved H-modes in the all-tungsten ASDEX Upgrade with nitrogen seeding. Nuclear Fusion, 51(11):113003, November 2011.

[50] G.F. Matthews. Plasma operation with an all metal first-wall: Comparison of an ITER-like wall 
with a carbon wall in JET. Journal of Nuclear Materials, 438:S2-S10, July 2013.

[51] M.N.A. Beurskens, J. Schweinzer, C. Angioni, A. Burckhart, C.D. Challis, I. Chapman, R. Fischer, J. Flanagan, L. Frassinetti, C. Giroud, J. Hobirk, E. Joffrin, A. Kallenbach, M. Kempenaars, M. Leyland, P. Lomas, G. Maddison, M. Maslov, R. McDermott, R. Neu, I. Nunes, T. Osborne, F. Ryter, S. Saarelma, P.A. Schneider, P. Snyder, G. Tardini, E. Viezzer, and E. Wolfrum. The effect of a metal wall on confinement in JET and ASDEX Upgrade. Plasma Physics and Controlled Fusion, 55(12):124043, December 2013.

[52] A. Kallenbach, M. Bernert, R. Dux, L. Casali, T. Eich, L. Giannone, A. Herrmann, R. McDermott, A. Mlynek, H.W. Müller, F. Reimold, J. Schweinzer, M. Sertoli, G. Tardini, W. Treutterer, E. Viezzer, R. Wenninger, and M. Wischmeier. Impurity seeding for tokamak power exhaust: from present devices via ITER to DEMO. Plasma Physics and Controlled Fusion, 55(12):124041, December 2013.

[53] P a Schneider, E Wolfrum, M G. Dunne, R Dux, A Gude, B Kurzan, T Pütterich, S K. Rathgeber, J Vicente, A Weller, and R Wenninger. Observation of different phases during an ELM crash with the help of nitrogen seeding. In 40th EPS conference on plasma physics, volume 56, Helsinki, February 2014.

[54] S.K. Rathgeber, R. Fischer, S. Fietz, J. Hobirk, A. Kallenbach, H. Meister, T. Pütterich, F. Ryter, G. Tardini, and E. Wolfrum. Estimation of profiles of the effective ion charge at ASDEX Upgrade with Integrated Data Analysis. Plasma Physics and Controlled Fusion, 52(9):095008, September 2010. 